\title{
RASTREAMENTO DO CÂNCER DO COLO UTERINO EM MULHERES IN DÍGENAS MBYÁ-GUARANI
}

CERVICAL CANCER SCREENING IN MBYÁ-GUARANI INDIAN WOMEN

DETECCIÓN DE CÁNCER DE CUELLO UTERINO EN MUJERES INDÍGENAS MBYÁ-GUARANI

Léia Gonchoroski Machado ${ }^{1}$

Aniúsca Vieira dos Santos 2

Giovana Tavares dos Santos 3

Claudia Giuliano Bica ${ }^{4}$

Palavras-chave:
Gestão em Saúde. Política de Saúde:

Promoção da Saúde; Qualidade de Vida; Sistema Único de Saúde (SUS).

Keywords:

Uterine Cervical Neoplasms; Health of Indigenous Peoples; Female; Indigenous Culture; Ethnicity and Health.

Palabras clave: Neoplasias del Cuello Uterino; Salud de Poblaciones Indigenas; Femenino; Cultura Indigena; Origen Étnico y Salud.

Submetido: 20 de Dez. de 2019

Aprovado:

Autor(a) para Correspondência: Claudia Giuliano Bica R. Sarmento Leite, 245 Centro Histórico - Porto Alegre, RS. CEP: $90050-170$ E-mail: claudia@ufcspa.edu.br 04 de Nov. de 2020

\section{RESUMO}

O objetivo deste estudo foi analisar a adesão à coleta citopatológica e descrever o perfil dos exames de rastreamento do câncer do colo uterino realizados em mulheres indígenas Mbyá-Guarani do litoral norte do Rio Grande do Sul, no período de 2014 a 2018, para qualificar e direcionar a implementação de estratégias em saúde nas políticas públicas voltadas à mulher indígena. Trata-se de um estudo transversal com abordagem quantitativa, com inclusão de dados dos registros dos exames de rastreamento de câncer do colo uterino disponiveis no Sistema de Informação do Câncer (SISCAN). Foram incluídas 113 mulheres Mbyá-Guarani, com idades entre 10 a 69 anos. A adesão à realização do exame citopatológico foi de $47 \%$ no período de 5 anos, com média etária de 29 anos. Em relação ao perfil citopatológico, $7 \%$ apresentaram alterações citopatológicas distribuídas em células atípicas de significado indeterminado (atypical squamous cells of undetermined significance [ASCUS]), possivelmente não neoplásicas, e lesão intraepitelial escamosa de baixo grau (low grade intraepithelial lesions [LSIL]) e $28 \%$ de desvio de flora, sugestivo de vaginose bacteriana, mais comumente associada à Gardnerella vaginalis. Os resultados demonstram que a adesão ficou abaixo da meta de $85 \%$ esperada pelo Ministério da Saúde (MS), evidenciando a necessidade de políticas públicas diferenciadas voltadas às populações de maior vulnerabilidade em saúde.

\footnotetext{
1. Aluna de Mestrado em Patologia na Universidade Federal de Ciências da Saúde de Porto Alegre (UFCSPA). E-mail: leia.gonchoroski85@gmail.com 0RCID: http://orcid.org/0000-0002-2900-8036

2. Aluna de Doutorado em Patologia na UFCSPA. E-mail: aniusca.vieira@gmail.com 0RCID: http://orcid.org/00000001-7468-591X

3. Doutora em Patologia pela UFCSPA. E-mail: santos.giovanat@gmail.com 0RCID: http://orcid.org/0000-0001$\underline{7364-4825}$

4. Doutora em Patologia pela UFCSPA. E-mail: claudia@ufcspa.edu.br ORCID: http://orcid.org/0000-0002-6763$\underline{6631}$
}

Certificação de redação científica: E.L.Freire Editora. Edição de texto: Evandro L. Freire. Revisão de provas: Texto definitivo lido e validado pelas autoras. 


\section{ABSTRACT}

This study aimed to analyze adherence to cytopathological examination and describe the profile of cervical cancer screening tests performed in Mbyá-Guarani Indian women from the northern coast of Rio Grande do Sul, Brazil, within the period from 2014 to 2018, in order to qualify and clarify the deployment of health strategies in public policy aimed at Indian women. This is a cross-sectional study with a quantitative approach, gathering data from the records of cervical cancer screening tests available in the Cancer Information System (SISCAN). The sample consisted of 113 Mbyá-Guarani women, aged from 10 to 69 years. Adherence to cytopathological examination reached $47 \%$ over a 5-year period, with an average age of 29 years. Regarding the cytopathological profile, $7 \%$ showed cytopathological changes distributed into atypical squamous cells of undetermined significance (ASCUS), possibly non-neoplastic, and low grade intraepithelial lesions (LSIL), and 28\% of flora deviation, suggesting bacterial vaginosis, most commonly associated to Gardnerella vaginalis. The results show that adherence was below the $85 \%$ goal set by the Ministry of Health $(\mathrm{MoH})$, highlighting the need for varied public policy approaches aimed at the most vulnerable populations from a health perspective.

\section{RESUMEN}

Este estudio tuvo como objetivo analizar la adherencia al examen citopatológico y describir el perfil de las pruebas de detección del cáncer de cuello uterino realizadas en mujeres indígenas Mbyá-Guaraní de la costa norte de Rio Grande do Sul, Brasil, en el período de 2014 a 2018, para calificar y aclarar el despliegue de estrategias de salud en las políticas públicas dirigidas a las mujeres indígenas. Se trata de un estudio transversal con enfoque cuantitativo, que recopila datos de los registros de pruebas de detección de cáncer de cuello uterino disponibles en el Sistema de Información del Cáncer (SISCAN). La muestra estuvo conformada por 113 mujeres Mbyá-Guaraní, de 10 a 69 años. La adherencia al examen citopatológico alcanzó el 47\% en un período de 5 años, con una edad promedio de 29 años. En cuanto al perfil citopatológico, el 7\% mostró cambios citopatológicos distribuidos en células atípicas de significado indeterminado (atypical squamous cells of undetermined significance [ASCUS]), lesiones posiblemente no neoplásicas y lesión intraepitelial escamosa de bajo grado (low grade intraepithelial lesions [LSIL]), y el $28 \%$ de desviación de la flora, sugiriendo vaginosis bacteriana, más comúnmente asociado a Gardnerella vaginalis. Los resultados muestran que la adherencia estuvo por debajo de la meta del $85 \%$ establecida por el Ministerio de Salud (MS), destacando la necesidad de enfoques variados de políticas públicas dirigidas a las poblaciones más vulnerables desde una perspectiva de salud.

\section{INTRODUÇÃO}

A Política Nacional de Atenção à Saúde dos Povos Indígenas, aprovada em 2002, caracterizase por complementar a saúde indígena no âmbito do Sistema Único de Saúde (SUS) mediante um modelo diferenciado de assistência à saúde que aborde suas especificidades culturais e étnicas via ações de prevenção de doenças, cuidados de saúde, acompanhamento das condições alimentares e nutricionais, bem como educação em saúde, entre outras iniciativas que contribuam para a proteção, promoção e recuperação da saúde das populações indígenas no Brasil ${ }^{1}$.

Entretanto, apesar da existência da regulamentação citada e da Política Nacional de Atenção Integral à Saúde da Mulher, aprovada em 2011 com vistas à redução da morbidade e mortalidade na população feminina, observa-se a necessidade de ampliar e qualificar as ações de assistência e promoção à saúde voltadas às mulheres indígenas e suas particularidades étnico-culturais ${ }^{2}$.

Autores afirmam que a incidência do câncer do colo uterino e de infecções sexualmente transmissíveis (IST) aumenta ano a ano em populações indígenas. Destacam-se entre os fatores de risco que favorecem a maior exposição e disseminação das IST e do câncer do colo uterino em mulheres indígenas: início precoce da vida sexual, múltiplos parceiros, multiparidade, escassez de informações sobre prevenção e cuidado, fator cultural e social e dificuldade de acesso aos serviços de saúde. Com isso, incluem-se essas populações entre aquelas de maior vulnerabilidade para o desenvolvimento dessas doenças ${ }^{3,4}$.

0 exame citopatológico é o principal método para o rastreamento do câncer do colo uterino, pois é por meio desse exame que as lesões são diagnosticadas logo no início, possibilitando seu tratamento 
precoce e um melhor prognóstico para a paciente. De acordo com o Instituto Nacional de Câncer (INCA), esse exame deve ser realizado periodicamente a cada 3 anos, por mulheres sexualmente ativas, após 2 exames anuais consecutivos com resultados negativos para lesões precursoras, pois a doença pode ser assintomática no início e apresentar rápida progressã $0^{5,6}$.

Segundo as políticas de prevenção do câncer do colo uterino e IST e do Plano de Ações Estratégicas para o Enfrentamento das Doenças Crônicas Não Transmissiveis (DCNT), espera-se obter $85 \%$ de adesão, enfocando as populações vulneráveis e de maior risco para o desenvolvimento de tais doenças, como as mulheres indígenas, pois um dos objetivos é a redução da mortalidade e das altas taxas de incidência dessa neoplasia ${ }^{7-9}$.

Nas últimas décadas houve um crescimento significativo da quantidade de informações sobre o perfil epidemiológico da saúde reprodutiva das mulheres, porém, ainda se observa escassez de dados sobre a saúde da mulher indígena brasileira. 0 pouco conhecimento dos determinantes do processo saúde-doença nas populações indígenas é o principal obstáculo para o direcionamento, a elaboração e a implementação de políticas públicas voltadas a um cuidado diferenciado e específico, pautadas pelos preceitos do SUS, das mulheres indígenas ${ }^{9-11}$.

Assim, este estudo teve por objetivo:

- Analisar a adesão à coleta citopatológica e descrever o perfil dos exames de rastreamento do câncer do colo uterino realizados em mulheres indígenas Mbyá-Guarani do litoral norte do Rio Grande do Sul, no período de 2014 a 2018, para qualificar e direcionar a implementação de estratégias em saúde nas políticas públicas voltadas à mulher indígena.

\section{METODOLOGIA}

Trata-se de estudo transversal com abordagem quantitativa sobre a adesão à coleta citopatológica e o perfil dos exames citopatológicos de mulheres indígenas, no período de 2014 a 2018. Os exames estão registrados no Sistema de Informação do Câncer, versão 1.6.0, do Ministério da Saúde (SISCAN WEB), uma versão em plataforma on-line que integra os Sistemas de Informação do Câncer do Colo do Útero (SISCOLO) e do Câncer de Mama (SISMAMA), cujas informações ficam disponíveis on-line em tempo real.

Para a análise quantitativa populacional de

\section{...ainda se observa escassez de dados sobre a saúde da mulher indigena brasileira.}

mulheres indígenas, a base de dados usada foi 0 cadastro de família das comunidades indígenas, registrado no Sistema de Informação de Saúde Indígena (SIASI), realizado pelos profissionais da saúde do Polo Base de Saúde Indígena de 0sório/ Distrito Sanitário Especial Indígena Interior Sul (DSEI/Interior Sul).

A população investigada é representada por mulheres Mbyá-Guarani com idades entre 10 e 69 anos que, durante o período de realização do estudo, residiam no litoral norte do Estado do Rio Grande do Sul (cidades de Osório, Caraá, Maquiné, Riozinho e Torres) distribuídas entre as comunidades indígenas: a) Sol Nascente (Osório); b) Varzinha (Caraá); c) Campo Molhado (Maquiné); d) Acampamento Som Dos - Solidão (Maquiné); e) Acampamento FEPAGRo (Maquiné); f) Km 45 e Km 50 (Riozinho); e g) Campo Bonito (Torres).

Para o estudo da adesão foi analisada a realização de pelo menos um exame durante o período estudado; para o estudo do perfil dos exames citopatológicos registrados no SISCAN caracterizamos os aspectos epiteliais e a microbiologia conforme a faixa etária. Os resultados de alterações citológicas foram classificados de acordo com o protocolo do Ministério da Saúde (MS), de 2016, e o Sistema Bethesda para Relato de Citologia Cervical, sendo descritas em conformidade com a Nomenclatura Brasileira para Laudos Cervicais e as condutas preconizadas pelo INCA e pela Sociedade Brasileira de Citopatologia $(\mathrm{SBC})^{7,12}$.

Para o estudo estatístico se usou o software SPSS, versão 25.0, empregando os testes de frequência e percentual, média e desvio padrão, além do quiquadrado com estimativas do intervalo de confiança (IC) de $95 \%$

Este estudo foi aprovado por meio do Parecer $n$. 3.000.589/2018 da Comissão Nacional de Ética em Pesquisa (CONEP) e do Parecer n. 2.930.088/2018 do Comitê de Ética em Pesquisa da Universidade 
Federal de Ciências de Saúde de Porto Alegre (CEP/UFSCPA), em conformidade com as Resoluções ns. 466/2012, $510 / 2016$ e 441/2011 do Conselho Nacional de Saúde (CNS) ${ }^{13-15}$.

\section{RESULTADOS}

Por meio do cadastro de família das comunidades indígenas estudadas, coletaram-se os dados de 113 mulheres Mbyá-Guarani com idades entre 10 e 69 anos (média etária = 29 anos; desvio padrão = 11,03), residentes no litoral norte do Rio Grande do Sul. Dentre essas mulheres, $47 \%$ aderiram à realização do exame de rastreamento do câncer do colo uterino. A distribuição anual das coletas citopatológicas é apresentada na Figura 1, conforme as faixas etárias. A adesão anual em todas as faixas etárias foi: $2014=13 \% ; 2015=23 \%$; $2016=34 \% ; 2017=23 \% ; 2018=8 \%$. Vale observar que a maior adesão ao exame ocorreu em $2016(34 \%)$ e a menor adesão veio no ano de 2018 (8\%).

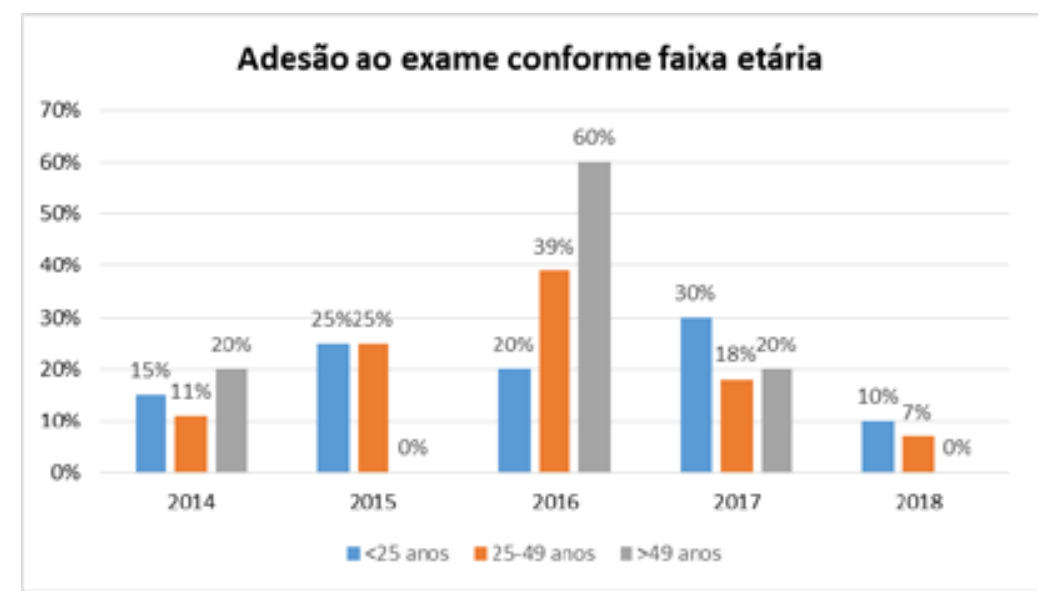

Figura 1. Adesão ao exame de rastreamento do câncer do colo uterino anualmente. Porto Alegre, 2019.

Fonte: Elaborada pelas autoras.

A distribuição de idade foi realizada de acordo com a faixa etária ( $<25$ anos, 25 a 49 anos, $>49$ anos), sendo que a maior adesão ao exame foi observada entre 25 a 49 anos (53\%), sintetizada na Tabela 1.

Tabela 1 - Distribuição da adesão ao exame citopatológico por faixa etária (2014-2018)

\begin{tabular}{|c|c|c|c|}
\hline Faixa etária & Total populacional & Total de adesão N (\%) & Total de não adesão $\mathrm{N}(\%)$ \\
\hline$<25$ & 68 & $20(38)$ & $48(80)$ \\
\hline $25-49$ & 40 & $28(53)$ & $12(20)$ \\
\hline$\geq 49$ & 5 & $5(9)$ & 0 \\
\hline Total & $113(100)$ & $53(47)$ & $60(53)$ \\
\hline
\end{tabular}

Fonte: Elaborada pelas autoras.

Durante o período estudado foram realizados 100 exames citopatológicos, sendo que dentre esses $13 \%$ apresentaram amostras insatisfatórias. Identificou-se a prevalência de alterações citopatológicas em $7 \%$ dos exames, distribuídos igualmente entre células atípicas de significado indeterminado (atypical squamous cells of undetermined significance [ASCUS]) e lesão intraepitelial escamosa de baixo grau (low grade intraepithelial lesions $[\mathrm{LSIL}])-3 \%$ cada $(p=0,436)$ (Tabela 2$)$. 
Tabela 2 - Distribuição anual da citologia cervical

\begin{tabular}{cccccc}
\hline Ano & $\begin{array}{c}\text { Critério normal } \\
\text { N (\%) }\end{array}$ & $\begin{array}{c}\text { Critério alterado } \\
\text { N (\%) }\end{array}$ & Total de exames & ASCUS N (\%) & LSIL N (\%) \\
\hline 2014 & $7(100)$ & 0 & 7 & 0 & 0 \\
2015 & $11(85)$ & $2(15)$ & 13 & $2(15)$ & 0 \\
2016 & $24(96)$ & $1(4)$ & 25 & $3(4)$ & $2(5)$ \\
2017 & $36(92)$ & 0 & 3 & 0 & 0 \\
2018 & $3(100)$ & $6(7)$ & 87 & $3(3)$ & $3(3)$ \\
\hline Total & $81(93)$ & & & 0 & $1(3)$ \\
\hline
\end{tabular}

Fonte: Elaborada pelas autoras.

$\mathrm{Na}$ análise microbiológica (Tabela 3 ) se encontrou a presença de desvio de flora sugestivo de vaginose bacteriana, mais comumente associada a Gardnerella vaginalis (28\%) e Trichomonas vaginalis ( $8 \%$ ). Ao longo do período estudado, a presença de Trichomonas vaginalis e/ou Gardnerella vaginalis foi observada em $67 \%$ das mulheres que repetiram o exame $(p=0,260)$. Não houve significância estatística entre a presença de agentes microbiológicos e alterações citopatológicas $(p=0,666)$, pois somente $33 \%$ das mulheres apresentaram concomitante Gardnerella vaginalis e alteração citopatológica.

Tabela 3 - Distribuição anual dos resultados da microbiologia

\begin{tabular}{|c|c|c|c|c|c|c|}
\hline Ano & $\begin{array}{l}\text { Total de } \\
\text { exames }\end{array}$ & $\begin{array}{c}\text { Lactobacilos } \\
\text { N (\%) }\end{array}$ & $\begin{array}{c}\text { Garderella } \\
\text { vaginalis N (\%) }\end{array}$ & $\begin{array}{c}\text { Trychomonas } \\
\text { vaginalis } \\
\mathrm{N}(\%)\end{array}$ & $\begin{array}{c}\text { Outros bacilos } \\
\text { N (\%) }\end{array}$ & $\begin{array}{l}\text { Cocos } \\
N(\%)\end{array}$ \\
\hline 2014 & 7 & $5(71)$ & $1(14)$ & 0 & 1 & 0 \\
\hline 2015 & 13 & $5(38)$ & $4(31)$ & 0 & 4 & 0 \\
\hline 2016 & 25 & $10(40)$ & $4(16)$ & $4(16)$ & 11 & 0 \\
\hline 2017 & 39 & $19(4)$ & $14(36)$ & $3(8)$ & 4 & $1(2)$ \\
\hline 2018 & 3 & $2(67)$ & $1(33)$ & 0 & 0 & 0 \\
\hline Total & 87 & $41(47)$ & $24(28)$ & $7(8)$ & $20(23)$ & $1(1)$ \\
\hline
\end{tabular}

Fonte: Elaborada pelas autoras.

\section{DISCUSSÃO}

Diante dos resultados observados ( $47 \%$ de adesão ao rastreamento do câncer do colo uterino), observa-se a diferença de $38 \%$ abaixo da meta esperada para o rastreamento em populações vulneráveis. Entretanto, a grande maioria (67\%) das mulheres Mbyá-Guarani que aderiram ao exame de papanicolau apresentaram uma microbiota sugestiva de infecções, como de desvio de flora sugestivo de vaginose bacteriana, mais comumente associado a Gardnerella vaginalis e/ou Trichomonas vaginalis. Esse fato é importante para o entendimento da proposição de políticas públicas assertivas voltadas a essa população, pois, além de campanhas para rastreamento do câncer do colo uterino se deve investir em políticas públicas que contemplem a prevenção de IST em indígenas.

Com o percentual de $47 \%$ de adesão ao exame de rastreamento do câncer do colo uterino no período de 5 anos, este estudo aponta que a cobertura não atingiu a meta de $85 \%$ estabelecida pelo MS $^{8}$ para população de risco. 0s dados corroboram com outro estudo ${ }^{16}$, que aponta uma adesão de $26,9 \%$ entre as mulheres indígenas de Queensland, na Austrália; por sua vez, outros pesquisadores ${ }^{17}$, que trabalharam com mulheres indígenas da região norte do Brasil, evidenciaram que a maioria dos exames é realizada por mulheres acima de 25 anos, distribuindo-se proporcionalmente entre as faixas etárias analisadas ${ }^{16,17}$.

Mesmo com a existência de políticas públicas de assistência à saúde da mulher, como as Diretrizes Brasileiras de Rastreamento do Câncer do Colo do Útero e os Protocolos da Atenção Básica: Saúde das Mulheres ${ }^{5,7}$, 
do Ministério da Saúde, sugere-se que as ações preventivas de saúde voltadas às mulheres indígenas devem contemplar sua realidade étnica e cultural.

Identificou-se aumento gradativo de coletas citopatológicas até o ano de 2016, seguido de significativa diminuição em 2018, fato que pode ser atribuído à troca da equipe de saúde indígena e à rotatividade de profissionais. Logo, as ações de promoção da saúde, no sentido de sensibilização para a realização do exame preventivo, exigem práticas educativas contínuas que contemplem e valorizem a visão cultural desses povos, bem como o fortalecimento do vínculo dos profissionais que cuidam dessas mulheres. Assim, diante do processo saúde-doença, faz-se necessária a participação coletiva dos profissionais da saúde, da sociedade e do Estado.

Em relação à prevalência de citologia cervical alterada, apesar de identificarmos $7 \%$ de alterações cervicais em indígenas Mbyá-Guarani, a literatura mostra um amplo intervalo de prevalência entre diferentes comunidades indígenas - de $3,3 \%$ a $22 \%{ }^{4,16,18-20}$.

Em outro estudo ${ }^{4}$, realizado em comunidades indígenas do Caaguazú, no Paraguai, identificouse prevalência de $13,18 \%$ de lesões precursoras do câncer do colo do útero, com maior frequência em mulheres indígenas Mbyá-Guarani, sendo superior à identificada no presente estudo. Uma hipótese é que os fatores comportamentais podem influenciar as taxas de prevalência, assim como o contato com não indígenas, a localização das comunidades e o precário acesso aos serviços de saúde ${ }^{4,20,21}$.

Além disso, a repetição do exame ocorreu principalmente em mulheres com a presença de Trichomonas vaginalis e/ou desvio de flora sugestivo de vaginose bacterina, comumente associado a Gardnerella vaginalis. Contudo, sabe-se que as infecções vaginais se encontram entre as causas mais frequentes da procura pela mulher por atendimento ginecológico devido à sua sintomatologia22,23. Em vista disso, as altas prevalências desses agentes microbianos nas mulheres indígenas podem estar associadas às suas particularidades socioculturais, tais como contato sexual precoce e múltiplos parceiros, fatores associados à persistência do vírus HPV e ao desenvolvimento de câncer do colo uterino $0^{12,23,24}$.

Nesse contexto, a concepção da mulher indígena sobre autocuidado e prevenção de agravos à saúde pode influenciar a adesão ao exame, visto que, para

\section{...a concepção da mulher indigena sobre autocuidado $e$ prevenção de agravos à saúde pode influenciar a adesão ao exame...}

ela, essas práticas se encontram em um segundo momento e que a doença ocorre somente a partir de uma sintomatologia ${ }^{20,25}$. 0 entendimento dessa realidade e de suas particularidades diante de sua compreensão do processo saúde-doença, hábitos e crenças facilitaria compartilhar o conhecimento do autocuidado e da saúde coletiva, respeitando a realidade e os costumes das diferentes culturas.

Diante disso, ressalta-se que a educação em saúde, ao promover reflexões críticas, em nível individual ou coletivo, valoriza os conhecimentos e as especificidades culturais para que, consequentemente, ocorra a adesão às práticas educativas em saúde e surjam programas de rastreamento de doenças, alcançando-se o bemestar físico e espiritual, além da equidade e da integralidade assistencial ${ }^{26,27}$.

A compreensão das particularidades e das peculiaridades culturais por parte dos profissionais da saúde que cuidam das mulheres indígenas MbyáGuarani se mostra de suma importância para a implementação de ações de prevenção de agravos e promoção da saúde, relacionando o atendimento aos costumes e às crenças, o que facilita o vínculo e a aproximação. Só assim, combinando esforços, podese atingir as metas e melhorar o status de saúde das populações vulneráveis.

\section{CONCLUSÃO}

Diante dos resultados deste estudo e das políticas públicas de saúde indígena atualmente existentes no Brasil, acredita-se ser necessário reformular as práticas de saúde voltadas a essa população, considerando sua concepção, seus saberes e suas crenças diante do processo de saúde-doença.

Como limitações deste estudo, destacase a precariedade de informações sobre a saúde sexual e reprodutiva das mulheres indígenas que viabilizem a avaliação de seu perfil de saúde. 
Contudo, a dificuldade de análise e comparação dos resultados observados, principalmente devido a laudos incompletos ou indisponíveis, evidencia a necessidade de mais estudos e dados quantitativos para o direcionamento de ações continuadas de assistência e avaliação dos serviços e dos programas de saúde.

Ao longo dos anos, as comunidades indígenas passaram por modificações em seu modo de vida que influenciaram negativamente seu status de saúde, com aumento da prevalência de doenças preveniveis, principalmente devido ao maior contato com a população não indígena. A atenção à saúde, mediante um modelo direcionado a essa população, e a interculturalidade são fatores de suma importância para alcançar melhores indicadores de saúde entre as mulheres indígenas. Além disso, faz-se necessário o reconhecimento da vulnerabilidade das populações indígenas e dos fatores que influenciam o processo saúde-doença, a fim de reformular as políticas públicas existentes conforme a real necessidade dessas mulheres. A partir disso, a participação indígena tende a aumentar nas ações de saúde e, consequentemente, seu vínculo com os profissionais e os gestores da saúde pode tornar-se cada vez mais forte, com vistas a garantir a proteção dessa população.

\section{CONTRIBUIÇÃO DAS AUTORAS}

Léia Gonchoroski Machado, Aniúsca Vieira dos Santos e Claudia Giuliano Bica contribuíram com a realização da pesquisa, o delineamento do estudo e a redação e revisão do manuscrito. Giovana Tavares dos Santos contribuiu com o delineamento do estudo e a redação e revisão crítica do manuscrito.

\section{REFERÊNCIAS}

1. Brasil. Política Nacional de Atenção à Saúde dos Povos Indígenas. Brasília (DF): Ministério da Saúde; 2015.

2. Brasil. Política Nacional de Atenção Integral à Saúde da Mulher: princípios e diretrizes. Brasília (DF): Ministério da Saúde; 2011.

3. Aguiar Jr PN, Stock GT, Lopes Jr GL, Almeida MS, Tadokoro H, Gutierres BS, et al. Disparidades na epidemiologia e no tratamento de câncer nas populações indígenas brasileiras. Einstein [serial on the internet]. 2016 [cited 2018 Sep 24];14(3):3307. Available from: https://doi.org/10.1590/S167945082016A03754
4. Velázquez C, Kawabata A, Rios-Gonzáles CM. Prevalencia de lesiones precursoras de cáncer de cuello uterino y antecedentes sexuales/ resproductivos de indígenas de Caaguazú, Paraguay 2015-2017. Rev Salud Pública Parag [serial on the internet]. 2018 [cited 2019 0ct 3];8(2):15-20. Available from: http://revistas.ins.gov.py/index. php/rspp/article/view/513

5. Brasil. Protocolos da atenção básica: saúde das mulheres. Brasília (DF): Ministério da Saúde; 2016.

6. Da Silva LR, Almeida CAPL, Sá GGM, Moura LKB, Araújo ETH. Educação em saúde como estratégia de prevenção do câncer do colo do útero: revisão integrativa. Revista Prevenção em Infecção e Saúde [serial on the internet]. 2017 [cited 2019 0ct 3];3(4):35-45. Available from: https://revistas.ufpi. br/index.php/nupcis/article/view/6708/pdf

7. Brasil. Diretrizes brasileiras para o rastreamento do câncer do colo do útero. Rio de Janeiro: Instituto Nacional de Câncer; 2016.

8. Brasil. Plano de ações estratégicas para - enfrentamento das doenças crônicas não transmissíveis (DCNT) no Brasil, 2011-2022. Brasília (DF): Ministério da Saúde; 2011.

9. Silva MAS, Teixeira BEM, Ferrari RAP, Cestari MEW, Cardelli AAM. Fatores relacionados à não adesão à realização do exame de papanicolau. Rev Rene [serial on the internet]. 2015 [cited 2019 Sep 18];16(4):532-9. Available from: https://www. redalyc.org/pdf/3240/324041519010.pdf

10. Costa PCG, Barros SRN, Pereira SMP, Gasque KCS, Galvão RS. Estudo epidemiológico do papilomavírus humano em populações indígenas do Brasil [document on the internet]. 2020 [cited 2020 out 10];1:20 33. Available from: https://www.arca.fiocruz.br/ bitstream/icict/43271/2/Cap Estudo epidemiológico do Papilomavírus humano em populações indígenas do Brasil.pdf

11. Fonseca AJ, Taeko D, Chaves TA, Amorim LDC, Murari RSW, Miranda $A E$, et al. HPV infection and cervical screening in socially isolated indigenous women inhabitants of the Amazonian rainforest. PLOS One [serial on the internet]. 2015 [cited 2019 Jul 10];10(7):e0133635. Available from: https:// www.sboc.org.br/images/downloads/2015 plos one hpv nas indigenas.pdf

12. Toninato LGD, Irie MMT, Consolaro MEL, Teixeira JJV, Boer CG. Vaginose bacteriana diagnosticada em exames citológicos de rotina: prevalência e características dos esfregaços de papanicolaou. RBAC [serial on the internet]. 2016 [cited $20200 \mathrm{ct}$ 14];48(2):165-9. Available from: http://www.rbac. org.br/artigos/vaginose-bacteriana-diagnosticadaem-exames-citologicos-de-rotina-prevalencia-ecaracteristicas-dos-esfregacos-de-papanicolaou48n-2/ 
13. Brasil. Resolução n. 466, de 12 de dezembro de 2012 [document on the internet]. Brasilia (DF): Ministério da Saúde [serial on the internet]. 2012 [cited 2019 Jun 28]. Available from: http://conselho. saude.gov.br/resolucoes/2012/Res0466.pdf

14. Brasil. Resolução n. 510, de 7 de abril de 2016. Dispõe sobre as normas aplicáveis a pesquisas em ciências humanas e sociais [document on the internet]. 2016 [cited 2019 Jun 25]. Available from: http://bvsms.saude.gov.br/bvs/saudelegis/ cns/2016/res0510 $07 \quad 04 \quad 2016 . h t m l$

15. Brasil. Resolução n. 441, de 12 de maio de 2011. Dispõe sobre as diretrizes para análise ética de projetos de pesquisas que envolvam armazenamento de material biológico humano ou uso de material armazenado em pesquisas anteriores [document on the internet]. 2011 [cited 2019 Jun 25]. Available from: https://conselho.saude.gov.br/ resolucoes/2011/Reso441.pdf

16. Whop LJ, Baade P, Garvey G, Cunningham J, Brotherton JML, Lokuge K, et al. Cervical abnormalities are more common among indigenous than other Australian woman: a retrospective record linkage study, 2000-2011. PLOS One [serial on the internet]. 2016 [cited 2020 0ct 10];11(4):e0150473. Available from: https://journals.plos.org/plosone/ article?id=10.1371/journal.pone. 0150473

17. Solé Pla MA, Corrêa FM, Claro IB, Da Silva MAF, Dias MBK, Bortolon PC. Análise descritiva do perfil dos exames citopatológicos do colo do úttero realizados em mulheres indígenas e não indígenas no Brasil, 2008-2011. Rev Bras Cancerol [serial on the internet]. 2012 [cited 2019 Nov 28]. 58(3):4619. Available from: https://rbc.inca.gov.br/revista/ index.php/revista/article/view/603

18. Fonseca AJ, Taeko D, Chaves TA, Amorim LDC, Murari RSW, Miranda AE, et al. HPV infection and cervical screening in socially isolated indigenous women inhabitants of the Amazonian rainforest. PLOS One [serial on the internet]. 2015 [cited 2019 May 21];10(7):e0133635. Available from: https://journals.plos.org/plosone/article/ authors?id=10.1371/journal.pone. 0133635

19. Speck NMG, Pinheiro JS, Pereira ER, Rodrigues D, Focchi GRA, Ribalta JCL. Rastreamento do câncer de colo uterino em jovens e idosas do Parque Indígena do Xingu: avaliação quanto à faixa etária preconizada no Brasil. Einsten [serial on the internet]. 2015 [cited 2019 May 21];13(1):52-7. Available from: https://www.scielo.br/scielo.php?pid=S1679$45082015000100010 \&$ script $=$ sci arttext\&tlng $=p t$

20. Freitas GV, Focchi GR, Pereira ER, Levi JE, Speck NM, Ribalta JC. HPV genotyping and p16 expressing in Xingu Indigenous Park, Brazil. Genet Mol Res [serial on the internet]. 2016 [cited 2019 Nov 15];15(3):5036840. Available from: https:// observatorio.fm.usp.br/handle/0PI/17278
21. Araújo MRA, Tavares MS, Souza VRFP, Bezerra D0. Saúde sexual e reprodutiva na etnia Xukuru do Ororubá: diga às mulheres que avancem. Saúde Debate [serial on the internet]. 2020 [cited 2019 Mar 18];44(124). Available from: https://www.scielosp. org/article/sdeb/2020.v44n124/193-204/pt/

22. Brasil. Protocolo clínico e diretrizes terapêuticas para atenção integral às pessoas com infecções sexualmente transmissíveis (IST). Brasília (DF): Ministério da Saúde; 2015.

23. Wohlmeister D, Vianna DRB, Helfer VE, Gimenes $F$, Consolaro MAEL, Barcellos RB, et al. Association of human papillomavirus and Chlamydia trachomatis with intraepithelial alterations in cervix samples. Mem Inst 0swaldo Cruz [serial on the internet]. 2016 [cited 20190 ct 11];(111):2. Available from: https://www.scielo.br/scielo.php?pid=S0074$\underline{02762016005002102 \& \text { script }=\text { sci arttext }- \text { c01 }}$

24. Rodrigues DA, Pereira ER, Oliveira LSS, Speck NMG, Gimeno SGGA. Prevalência de atipias citológicas e infecção pelo papilomavírus humano de alto risco em mulheres indígenas Panará, povo indígena do Brasil Central. Cad Saúde Pública [serial on the internet]. 2014 [cited 2019 Mar 26];30(12):2587-93. Available from: https://www.scielosp.org/article/csp/2014. v30n12/2587-2593/

25. Medina S, Sierra A, González EQ, Cruz SV, Jiménez $C V$, Amaya MP, et al. Intercultural and participative strategy for prevention of cervical cancer in indigenous people. Eur J Public Health [serial on the internet]. 2020 [cited 2020 0ct 12];30(5). Available from: https://doi.org/10.1093/eurpub/ckaa166.753

26. Barbosa MIS, Bosi MLM. Vínculo: um conceito problemático no campo da saúde coletiva. Physis (Rio J) [serial on the internet]. 2017 [cited 2020 Oct 12];(27):1003-22. Available from: https://doi. org/10.1590/S0103-73312017000400008

27. Da Costa FAS, Catanio PAG, Aragão AEA, Ponte HMS, Fardin FP, Araújo LM. Práticas populares em saúde indígena e integração entre o saber científico e popular: revisão integrativa. Sanare (Sobral, Online) [serial on the internet]. 2016 [cited 2019 Mar 18];15(2):112-9. Available from: https://sanare. emnuvens.com.br/sanare/article/view/1045
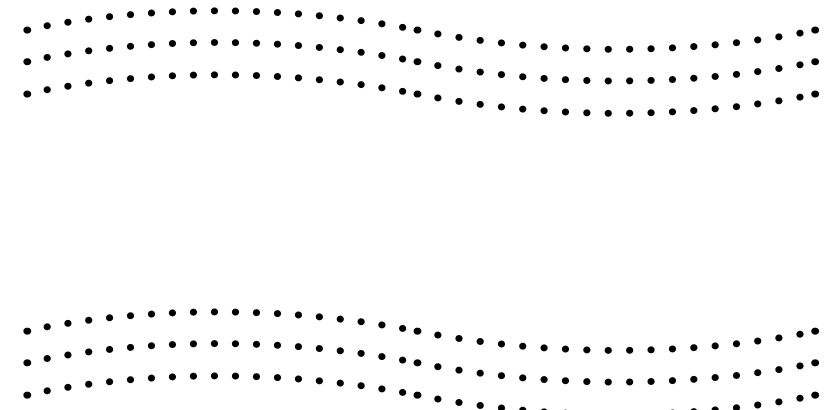